\title{
Alexitimia: Uma Revisão do Conceito
}

\author{
Berenice Victor Carneiro ${ }^{1}$ \\ Elisa Medici Pizão Yoshida \\ Pontificia Universidade Católica de Campinas
}

\begin{abstract}
RESUMO - Alexitimia é um termo empregado no diagnóstico clínico de pessoas com acentuada dificuldade ou incapacidade para expressar emoções e significa "sem palavras para as emoções". O objetivo deste artigo é apresentar uma revisão do conceito de alexitimia, enfatizando a importância das recentes contribuições da neurobiologia. É abordado o desenvolvimento do conceito desde os anos 70, ressaltando as contribuições de Sifneos e a estreita relação desse conceito com as doenças psicossomáticas. Em seguida, apresenta-se o conceito a partir de uma perspectiva multifatorial e destaca-se a importância do substrato neurológico para a melhor compreensão da etiologia. Finalmente, sugere-se ser necessária a compreensão integrada do papel dos fatores etiológicos para o tratamento e prevenção.
\end{abstract}

Palavras-chave: emoção; sintomas afetivos; distúrbios emocionais; ansiedade.

\section{A Review of Alexithymia Concept}

\begin{abstract}
Alexithymia is a term used for clinic diagnosis of those with marked difficulty in verbally expressing emotions, and it means "no words for emotions". The aim of this article is to present a review of the literature on the Alexithymia concept, highlighting additions from recent findings in neurobiology. It includes developments on the concept since the 70's, emphasizing the contributions of Sifneos and the straight association of this concept with psychosomatic diseases. Then, it presents the concept from a multifactor view indicating the role of neurobiology in order to increasingly understand its etiology. Finally, it stresses that integrating the etiological sources will enhance comprehension of treatment and prevention.
\end{abstract}

Keywords: emotions; affective symptoms; emotional disturbances; anxiety.

Este trabalho apresenta uma revisão da literatura sobre o conceito de alexitimia, com ênfase sobre as mudanças que este vem sofrendo desde sua proposição nos anos 70 , por Sifneos (1972/1977), até os dias atuais, quando os desenvolvimentos das neurociências trouxeram novos modos de compreensão sobre ele. Para tanto, inicia-se com referências às origens do conceito, em que a alexitimia era associada às doenças psicossomáticas, menciona-se as formas de tratamento e, em seguida, apresenta-se uma visão mais atual do conceito, que passou a ser entendido a partir de uma perspectiva multifatorial.

O termo alexitimia foi sugerido por Sifneos para se referir àqueles pacientes com uma vida emocional pobre em sonhos e fantasias e que demonstravam não ter palavras para nomear ou expressar as emoções (Sifneos, 1972/1977, 1991). Um pouco antes, em 1963, um conceito semelhante havia sido proposto por Marty e M'Uzan, iniciadores do movimento científico que deu origem à chamada Escola Psicossomática de Paris (Peçanha, 1998). Tratava-se do pensamento operatório, construto desenvolvido para designar um estilo de raciocínio concreto, objetivo, voltado para a realidade externa, com uma vida interior pobre e com ausência de reação afetiva frente a situações de perda ou traumas (Marty \& M'Úzan, 1963/1994).

Apesar de ambos os construtos, alexitimia e pensamento operatório, terem sido inicialmente associados a pacientes psicossomáticos e muitas vezes empregados de forma alter-

1 Endereço: Rua Dona Libânia, 1985, Ap 91. Campinas, SP. CEP 13015-090. E-mail: berenice_carneiro@hotmail.com. nada na literatura (Silva \& Caldeira, 1992), foram concebidos segundo vertentes teóricas distintas. Sifneos, um psicanalista de orientação freudiana, considerava que as doenças psicossomáticas diferiam das neuroses, neuroses narcísicas e psicoses, quanto à sua natureza (Messer \& Warren, 1995). Enquanto estas últimas se enquadrariam entre as doenças mentais, propriamente ditas, e seus sintomas se caracterizariam por uma expressão simbólica de conflitos psíquicos infantis, as afecções psicossomáticas se aproximariam das neuroses atuais, propostas por Freud, cujos sintomas seriam "principalmente de ordem somática" (Laplanche \& Pontalis, 1967/1970, p.383).

Quanto ao conceito de pensamento operatório, faz parte de um arcabouço teórico no qual "a palavra psicossomática agrupa dois termos, sem traço de união entre eles, a fim de conotar a unidade fundamental entre o psíquico e o corpo" (Peçanha, 1998, p.130). Trata-se, portanto, de um conceito que parte de uma concepção monista de homem, de acordo com a qual, o soma e o psiquismo só são divididos por uma questão de ênfase, sendo inadequado falar exclusivamente em sintomas físicos ou somáticos. De acordo com essa perspectiva, a unidade essencial do organismo humano decorre da hierarquização progressiva de todas as funções que participam de sua organização e inclui as noções de: mentalização (em um nível superior), depressão essencial, pulsão de morte, doença somática (em um nível mais inferior), entre outras. Todas elas, segundo Peçanha, devem ser consideradas na definição de pensamento operatório, que fica sem sentido se descontextualizada teoricamente. 
Houve, portanto, um descontentamento por parte daqueles que adotavam a concepção monista do organismo humano com o uso frequente na literatura do termo pensamento operatório como sinônimo de alexitimia. As diferenças epistemológicas se refletiam, de forma substancial, tanto nas pesquisas quanto na prática clínica.

Durante as décadas de 80 e 90 , muitas pesquisas foram realizadas no sentido de se buscar uma melhor compreensão da etiologia e das dimensões da alexitimia. Porém, a falta de uma teoria consistente que desse suporte ao construto teórico foi um dos determinantes para as inconsistências e a falta de consenso encontradas na literatura (Larsen, Brand, Bermond \& Hijman, 2003; Taylor, 2000). Estudos empíricos, com amostras pequenas, delineamentos inadequados de pesquisa, utilização de instrumentos fracos metodologicamente (Lane, Sechrest, Riedel, Shapiro \& Kaszniak, 2000; Larsen \& cols., 2003; Morrison \& Pihl 1989; Taylor, 2000; Taylor, Ryan \& Bagby, 1985; Taylor \& Bagby, 2004), imprecisão na definição e operacionalização do construto (Bermond, 2003; Larsen \& cols., 2003), entre outros, parecem ser também outras razões para a falta de consenso. Como uma das implicações dessas diferenças epistemológicas para a prática clínica, destaca-se aqui a forma de tratar os chamados pacientes psicossomáticos.

A proposta do Instituto de Psicossomática de Paris (Psychosomatic Institute of Paris-IPSO) é a de compreender e tratar doenças somáticas segundo o postulado fundamental de que o processo de somatização aparece quando o sujeito não é capaz de tratar, mentalmente, as contradições que pesam sobre ele (Peçanha, 1998). Por outro lado, os autores americanos, entre eles Sifneos, na medida em que a alexitimia não teria necessariamente um significado simbólico para o sujeito, defendem a adoção de uma postura didática por parte do terapeuta, a fim de ajudar o paciente a aprender a reconhecer e discriminar suas emoções. Para estes últimos autores, a aplicação da técnica padrão da psicanálise a alexitímicos não é vista como eficaz (Taylor, 1990). Esse enfoque, provavelmente, favoreceu a aceitação do conceito de alexitimia por autores de outras vertentes teóricas da psicologia, entre os quais os cognitivistas, os psicodinâmicos e os integracionistas.

Considerada, inicialmente, um traço de personalidade com implicações em transtornos psiquiátricos e doenças orgânicas (Bagby, Taylor \& Parker, 1994; Martínez-Sánchez, Ato-Garcia \& Ortiz-Soria, 2003; Taylor, 1984), características alexitímicas foram também identificadas em populações clínicas e não clínicas, não estando necessariamente associadas a distúrbios mentais específicos ou psicossomáticos (Buchanan, Waterhouse \& West Jr., 1980; Taylor \& Bagby, 2004). Ultimamente, a definição mais encontrada na literatura é a de que se trata de um construto multidimensional, integrado pelos seguintes fatores: (a) dificuldades em identificar e descrever sentimentos subjetivos; (b) dificuldades em fazer distinção entre emoções e sensações físicas; (c) escassez de sonho e incapacidade de simbolizar ou fazer relação entre afeto e fantasia (Sifneos, 1991); e (d) um estilo de raciocínio concreto e objetivo, voltado para a realidade externa (Taylor, 1984; Taylor \& Bagby, 2004). Não há, no entanto, consenso na literatura quanto à importância de cada fator nessa última definição. Alguns autores enfatizam mais os aspectos cognitivos da alexitimia do que os emocionais, como é o caso de Taylor e cols. (1994; 2004). Essa crítica já havia sido expressa, por exemplo, por Haviland e Reise (1996) e, posteriormente, por Larsen e cols. (2003).

\section{Etiologia da Alexitimia}

Em relação à etiologia, Sifneos, Apfel-Savitz e Frankel (1977), no início de seus estudos, já apontavam para uma variedade de fatores etiológicos da alexitimia, tais como: genéticos, fisiológicos, neuroanatômicos, psicossociais, assim como alterações neuroquímicas e de desenvolvimento. Posteriormente, Sifneos (1991) veio a propor que as várias etiologias fossem classificadas segundo dois tipos: as de origem biológica e as de causa psicossocial (ou de desenvolvimento). E, seguindo a tendência dos pesquisadores europeus (e.g., Pedinielli \& Rouan, 1998), identificou como alexitimia primária as do primeiro tipo, e como alexitimia secundária aquelas com causa psicossocial.

Alexitimia primária seria a forma biológica da doença, advinda de defeito estrutural neuroanatômico ou deficiência neurobiológica e pressupõe a interrupção da comunicação entre os dois hemisférios cerebrais ou entre o sistema límbico e o córtex (Sifneos, 1991; Taylor, 1984). Para fundamentar essa hipótese, vários estudos reportam alexitimia em pacientes com disfunção cerebral evidente. Buchanan e cols. (1980) mencionam o estudo de Hoppe e Bogen (1977), no qual 12 pacientes epiléticos, após serem submetidos à comissurotomia completa (seç̧ão do corpo caloso e comissura anterior), apresentaram características da alexitimia.

A alexitimia primária também costuma ser considerada como um traço de personalidade, devido ao seu caráter mais duradouro (Pedinielli \& Rouan, 1998). Essa interpretação tem sido usada, por exemplo, em estudos que buscam associação entre altos níveis de alexitimia e quadros de dependência de substâncias psicoativas e de alcoolismo, nos quais a alexitimia funcionaria como um fator de risco (Corcos \& Jeammet, 2000; Maciel \& Yoshida, 2006). Apesar de não haver concordância entre os estudos quanto aos índices de prevalência de alexitimia entre dependentes de álcool (as estimativas variam entre $35,8 \%$ a $78 \%$ ), há indicações de que a alexitimia primária ocuparia posição central em quadros de alcoolismo (Taieb \& cols., 2002).

No caso da alexitimia secundária, Sifneos (1991) se refere a uma reação aos efeitos de traumas ou doenças sérias, podendo ter como origem situações traumáticas vividas em períodos críticos do desenvolvimento infantil ou traumas intensos na idade adulta. As experiências traumáticas, tanto na infância como na idade adulta, podem ser de tal magnitude que levem a alterações estruturais do funcionamento psíquico, afetando, principalmente, o componente afetivo das emoções, com implicações significativas para a vida do indivíduo (Sifneos, 1991; Taylor, 1984). Acredita-se que, quando as situações traumáticas ocorrem antes do desenvolvimento da linguagem, há posteriormente prejuízo na habilidade de usar as palavras para expressar os sentimentos. Sifneos explica que, como consequência, as emoções seriam expressas em termos de sensações somáticas ou reações comportamentais, em vez de relacionadas a pensamentos. 
Ainda em relação aos traumas vividos na infância, Krystal, Giller e Cicchetti (1986) defendiam que aqueles decorrentes de excessos ou privações nas relações mãe-criança impediriam o desenvolvimento adequado da capacidade de expressão afetiva, assim como da função simbólica. Como consequência, a falha na internalização da função parental de proteção, o uso excessivo da negação e repressão de afetos, o colapso dos mecanismos de defesa do ego, entre outros, levariam a uma paralisação do desenvolvimento afetivo normal, podendo resultar nos transtornos psicossomáticos.

Também com base em sua experiência como psicanalista, Mc Dougall (1982) dá ênfase às vivências emocionais primárias entre mãe-filho e sua associação com a alexitimia secundária. Carências afetivas na primeira infância, decorrentes de comprometimentos no vínculo materno, revelam-se em um processo primitivo e infra-verbal, como uma forma regredida de reação. Para ela, seria então um mecanismo defensivo contra o surgimento de ansiedades psicóticas ligadas, por exemplo, ao perigo de perda de identidade. De acordo com essas hipóteses, pessoas alexitímicas descarregam na ação ou no ambiente suas expressões emocionais, separando-as de sua subjetividade e demonstrando dificuldade de contato com sua realidade psíquica.

No que se refere à associação entre alexitimia e os traumas intensos vividos de forma abrupta na idade adulta, também há concordância entre os pesquisadores. Experiências traumáticas como as decorrentes de guerra, abuso físico, desastre natural, sequestro, doença terminal, entre outras, levariam indivíduos, sem características alexitímicas, a sofrer uma regressão na função afetivo-cognitiva e a reagir à situação de forma a impedir a consciência da emoção (Krystal \& cols., 1986; Silva \& Caldeira, 1992; Taylor, 1984). Nas palavras de Sifneos (1991), o estresse intenso levaria a um "embotamento dos sentimentos", evitando a consciência das emoções e expressão dos sentimentos (p.119). Para Campbell (1940/1996), essa forma de alexitimia caracteriza-se por um estado, uma reação ou forma de defesa para impedir os efeitos da doença ou trauma sobre a vida afetiva e emocional da pessoa, evitando assim a depressão ou a dor. Dentro dessa perspectiva, "a alexitimia secundária não aparece necessariamente vinculada a uma patologia, mas funciona como uma estratégia de enfrentamento desenvolvida pelo indivíduo, frente a uma situação conflituosa e de difícil resolução" (Maciel \& Yoshida, 2006, p.44).

Além desses fatores intrapsíquicos e de desenvolvimento, Haviland, Warren e Riggs (2000), Lesser (1981) e Taylor (1984) apontam ainda para a possibilidade de associação entre fatores socioculturais e alexitimia. Em sua revisão da literatura, Taylor cita os estudos de Leff em 1973, e de Borens, Groose-Schultze, Jaensch e Kortemme em 1977, que demonstraram que estilos de comunicação característicos de uma cultura, nível educacional ou classe socioeconômica poderiam impor características ou limites à verbalização das emoções. Por exemplo, Taylor menciona um estudo em que pacientes psicossomáticos, de nível socioeconômico mais baixo, usaram menos palavras para as emoções e demonstraram ter menos fantasias. Haviland e cols., em seu estudo, encontraram correlação fraca, porém negativa e estatisticamente significativa, entre alexitimia e nível educacional.
Estudos brasileiros corroboram estas observações. Por exemplo, gestantes atendidas pelo Sistema Único de Saúde (SUS) apresentaram escores médios muito superiores aos de estudantes universitários (Yoshida, 2000, 2007; Yoshida \& Silva, 2007), quando avaliadas com a Toronto Alexithymia Scale (TAS-26).

Resultados semelhantes são mencionados na pesquisa com estudantes brasileiros, realizada por Fortes (1994, citado em Oliveira, 2001,) para adaptação da versão da TAS-20. Em outro estudo brasileiro de validação da TAS-20, Wiethaeuper, Balbinotti, Pelisoli e Barbosa (2005) também fazem referência às implicações socioculturais sobre a expressão de alexitimia. Deve-se observar, todavia, como explica Oliveira, que a hipótese sobre a interferência do nível educacional ou classe socioeconômica nas manifestações da alexitimia ainda é um tema controverso, e que precisa continuar a ser investigada por meio de instrumentos adaptados às características socioculturais da população pesquisada.

\section{Contribuições da Neurobiologia}

Como indicado anteriormente, as pesquisas partiram de um referencial clínico, mas evidências acumuladas indicam que a alexitimia pode ser observada tanto em populações clínicas como não clínicas, não estando associada diretamente a distúrbios mentais específicos ou psicossomáticos. Esses achados vêm impulsionando novas pesquisas na área da neurobiologia, como forma de melhor compreender a etiologia da alexitimia.

Recentemente, à luz de exames modernos de neuroimagem (por exemplo, a ressonância magnética funcional e a tomografia por emissão de pósitrons), foi possível uma reavaliação da hipótese biológica inicial de alexitimia, levantada por Sifneos na década de 70. Estudos com populações clínicas e não-clínicas têm sugerido que a alexitimia é uma expressão do funcionamento neurológico, constituída de um conjunto de características afetivas e cognitivas que reflete variações na organização e funcionamento do cérebro (Taylor \& Bagby, 2004; Larsen \& cols., 2003; Taylor, 2000).

A informação emocional e não-verbal é normalmente processada no hemisfério direito (HD), havendo evidência de sua função excitatória e de mediação das respostas autonômicas aos estímulos emocionais (Taylor, 2000). Por outro lado, o processamento da informação verbal ocorre no hemisfério esquerdo (HE), que exerce papel de controle inibitório sobre a função excitatória do HD (Bermond, 2003; Larsen \& cols., 2003; Taylor, 2000). Na alexitimia, parece haver uma dissociação entre os dois hemisférios, o que afetaria a modulação afetiva, a capacidade de comunicar afetos, a capacidade para fantasiar e elaborar as tensões internas e angústias (Oliveira, 2001).

Com base em estudos com pacientes que sofreram alguma lesão, ou apresentavam disfunção em determinadas áreas corticais pré-frontais do hemisfério direito, ou do corpo caloso, Bermond (2003) propôs uma nova categorização para alexitimia, distinguindo a de Tipo I e de Tipo II. Na alexitimia Tipo I, tanto os componentes afetivos, quanto os cognitivos, das experiências emocionais estariam comprometidos. Por outro lado, na alexitimia Tipo II, apenas os componentes 
cognitivos das emoções estariam prejudicados. Nesse caso, a alexitimia estaria associada às doenças psicossomáticas, como será mais bem explicado posteriormente.

Considera-se alexitimia Tipo I quando há lesão: (1) no hemisfério direito; (2) em estruturas pré-frontais como o córtex cingulado anterior (CCA), região dorsolateral e órbito-frontal; ou ainda, (3) se houver hipoatividade nessas regiões pré-frontais (Larsen \& cols., 2003). Essas regiões pré-frontais são ricas em receptores dopaminérgicos, têm conexão neuronal com o sistema límbico (motivacional) e, quando lesionadas ou hipoativas, comprometem a vivência e expressão das emoções (Bermond, 2003). Nesse tipo de alexitimia, o componente afetivo (valor ou valência) das experiências emocionais e as respostas fisiológicas correspondentes ficariam comprometidos, uma vez que estruturas que exercem papel fundamental no processo de avaliação da emoção estariam prejudicadas. Como consequência, o processo de elaboração cognitiva das experiências emocionais também ficaria afetado (Bermond, 2003; Larsen \& cols., 2003).

De acordo com documento produzido pela Organização Mundial da Saúde (World Health Organization-WHO), as regiões órbito-frontal e do CCA têm sido associadas a situações de aprendizagem instrumental (estímulos reforçadores e punições) que incluem emoções (WHO, 2004). O CCA tem papel importante na percepção dos sentimentos, pois mantém a informação na memória operacional, enquanto auxilia na organização dos aspectos fisiológicos e motores do sistema de resposta emocional (Taylor, 2000). O CCA é uma pequena região localizada na parte anterior do corpo caloso que liga os lobos temporais entre si e à amígdala (Lane \& cols., 2000; Larsen \& cols., 2003). A amígdala, por sua vez, faz parte do sistema límbico que tem ligação com os gânglios de base - região abundante em neurônios dopaminérgicos, com controle sobre os movimentos e papel importante nas emoções. Quando esse circuito (estruturas pré-frontais específicas, sistema límbico e gânglios de base) se encontra disfuncional, há prejuízo das emoções, tomada de decisão, planejamento e iniciativa (WHO, 2004).

O estudo de Lane e cols. (2000) envolveu pacientes alexitímicos, com ausência tanto da vivência (experiência) emocional quanto de seu processamento cognitivo. Por meio de exames de neuroimagem, os resultados do estudo sugeriram que a incapacidade de regular e processar cognitivamente as emoções estava associada a um déficit na atividade do CCA direito, durante excitação emocional. Lane e cols. atribuem a essa área a habilidade de perceber o estado mental e emocional, tanto em si quanto no outro, sendo que a existência de lesões afetaria os componentes afetivos e, por consequência, também os cognitivos.

Taylor e Bagby (2004) apontam que a hipótese de Lane e cols. (2000) foi confirmada no estudo francês de Berthoz e colaboradores, em 2002, que usou o exame de ressonância magnética funcional para avaliar a atividade cerebral durante observação de cenas com conteúdo emocional. Em relação às atividades do CCA e região médio-frontal, foram observadas diferenças associadas ao valor emocional atribuído ao estímulo (cena com conteúdo emocional), uma tarefa cognitiva atribuída ao hemisfério esquerdo.
Na alexitimia Tipo II ou cognitiva, o problema seria decorrente de lesão ou disfunção no corpo caloso, feixe nervoso que comunica os dois hemisférios e permite integração e transmissão de informações entre eles. Pacientes que apresentam lesão nesse feixe nervoso demonstram comprometimento apenas no componente cognitivo das emoções (Larsen \& cols., 2003). Esses pacientes teriam a vivência emocional acompanhada das respostas autonômicas correspondentes, mas não conseguiriam interpretar ou elaborar cognitivamente a emoção vivida nem a excitação autonômica experienciada (Bermond, 2003). Essa hipótese foi confirmada em alguns estudos citados a seguir.

Bermond (2003) menciona, por exemplo, o estudo de Gazzaniga e LeDoux em 1978, em que pacientes com o corpo caloso trans-seccionado não tiveram dificuldades para atribuir valor a estímulos emocionais processados apenas no hemisfério direito (HD). Porém, a avaliação que faziam se restringia aos aspectos emocionais, sem a elaboração cognitiva dos mesmos, a qual seria uma função do hemisfério esquerdo. Esse estudo utilizou um delineamento de pesquisa que permitiu evidenciar o funcionamento de cada hemisfério e do corpo caloso.

Normalmente, quando um estímulo é apresentado no campo visual (direito e esquerdo) das pessoas, ele é processado de forma cruzada pelos hemisférios, e o corpo caloso possibilita a integração das informações processadas separadamente (Purves \& cols., 1997). No estudo de Gazzaniga e LeDoux (1978), descrito por Bermond (2003), os pesquisadores apresentaram informações apenas no campo visual esquerdo dos pacientes e estas foram então processadas diretamente no HD (não-verbal). Uma vez que as informações estavam impedidas de chegar ao HE devido à lesão, não eram elaboradas cognitivamente. Resultado semelhante foi observado por Buchanan e cols. (1980), quando estímulos eliciadores de emoção foram apresentados a uma paciente com corpo caloso seccionado. Nesse estudo, como no anterior, a paciente reagia demonstrando emoção, mas não conseguia explicar claramente o que lhe causava tal reação.

Para Bermond (2003), estudos como os citados acima evidenciam que, por falta de comunicação inter-hemisférica, os componentes afetivos dos estímulos emocionais são enviados diretamente do sistema límbico ao HE. Este último, não conseguindo interpretar adequadamente, nem inibir ou controlar as respostas fisiológicas autonômicas decorrentes da emoção vivida (uma função que depende de integração entre os dois hemisférios), faria inferências incorretas a respeito das causas das respostas fisiológicas autonômicas, interpretando-as como sintomas de doenças (Taylor \& Bagby, 2004). Larsen e cols. (2003) acrescentam que, quando as respostas autonômicas não são inibidas ou controladas, tornam-se desreguladas, crônicas e desencadeiam níveis elevados de adrenalina e noradrenalina no organismo. $\mathrm{O}$ sistema imunológico, enfraquecido, facilitaria a ocorrência das doenças “(psico)somáticas” (p. 538). Dessa perspectiva, portanto, a associação entre alexitimia Tipo II e doenças psicossomáticas pode ser sugerida pela hipótese de incapacidade de conscientemente regular ou modular as emoções, devido à falta de integração das informações processadas em cada hemisfério. 
Taylor e Bagby (2004) discutem vários estudos experimentais realizados recentemente, e um deles corrobora os achados sobre o funcionamento neurológico e o processamento das emoções. O estudo de Hariri, Bookheimer e Mazziotta (2000, conforme citado em Taylor \& Bagby, 2004), ao utilizar exame de neuroimagem, constatou aumento na atividade das amígdalas direita e esquerda quando os participantes tinham como tarefa emparelhar uma figura alvo a figuras de faces que expressavam raiva ou medo. Quando a tarefa era decidir entre duas palavras e nomear aquela que identificava a expressão observada em uma face (tarefa cognitivo-verbal), houve redução de atividade na amígdala e aumento de atividade no córtex pré-frontal direito. Como sugerem Taylor e Bagby, esses resultados ilustram o modo de funcionamento neuronal que permite aos indivíduos utilizarem funções corticais superiores (como decidir e nomear) para regular o processamento das emoções em regiões subcorticais do sistema límbico, como a amígdala. Acrescentam, ainda, que por meio dos resultados do referido estudo de Hariri e colaboradores, poder-se-ia levantar a hipótese de que indivíduos mais alexitímicos, portanto com menos capacidade para identificar e nomear suas emoções, teriam mais dificuldades em controlar as respostas da amígdala do que indivíduos menos alexitímicos.

\section{Considerações Finais}

Estudos em neurobiologia indicam que a modulação de respostas emocionais, a partir de sistemas corticais superiores, envolve uma integração das funções de cada hemisfério, ou seja, da capacidade verbal do hemisfério esquerdo com a capacidade de avaliar e regular as emoções do hemisfério direito. Apesar de promissores, há de se notar que esses estudos são ainda incipientes, posto que baseados em situações bastante controladas e específicas, nem sempre passíveis de generalização para situações complexas de vida em que inúmeros fatores podem comprometer a capacidade de entrar em contato com as emoções e verbalizá-las.

As novas evidências, propiciadas pelas pesquisas na área da neurobiologia, descortinaram uma nova perspectiva de exploração da alexitimia e vêm colaborando para o papel desempenhado pelos fatores biológicos na sua etiologia. No entanto, muitas questões seguem sem respostas e demandam novas pesquisas. É necessário, por exemplo, esclarecer o papel desempenhado pelos demais fatores que têm se mostrado relevantes na etiologia da alexitimia e compreender como eles interagem entre si, potencializando-se ou abrandando os seus efeitos.

Além disso, é preciso elucidar como cada um dos fatores contribui para outras comorbidades às quais a alexitimia tem sido associada, tais como, dependência de substâncias químicas (Carneiro, 2008; Haviland, Shaw, Cummings \& MacMurray, 1988; Maciel \& Yoshida, 2006; Mann, Wise, Trinidad \& Kohanski, 1995), altos níveis de ansiedade (Jula, Saminen \& Saarijãrvi, 1999; Pregnolatto, 2005), depressão (Luminent, Bagby \& Taylor, 2001), hipertensão essencial (Jula \& cols., 1999; Lipp, Alcino, Bignotto \& Malagris, 1998), dor pélvica (Oliveira, 2001), transtorno de pânico (Torres \& Crepaldi, 2002), estresse pós-traumático (Morrison \& Pihl, 1989), enfermidades inflamatórias intestinais (Guimarães \& Yoshida, 2008), entre outras.

Em face do valor heurístico que o conceito de alexitimia tem demonstrado, é preciso, portanto, seguir pesquisando, no intuito de esclarecer mais completamente sua natureza e com isto abrir perspectivas mais efetivas para o seu tratamento e prevenção.

\section{Referências}

Bagby, R. M., Taylor, G. J., \& Parker, J. D. (1994). The twentyitem Toronto Alexithymia Scale II. Convergent, discriminant, and concurrent validity. Journal of_Psychosomatic Research, $38,33-40$.

Bermond, B. (2003). Brain and Alexithymia. Retirado em 12/12/2005 de http:\\spitswww.uvt.nl/web/fsw/psychologie/ emotions2003/2/h11.pdf.

Buchanan, D. C., Waterhouse, G. J., \& West Jr., S. C. (1980). A proposed neurophysiological basis of alexitimia. Psychotherapy and Psychosomatics, 34, 248-255.

Campbell, R. J. (Ed.) (1996). Psychiatric Dictionary (7 ${ }^{\mathrm{a}}$ ed.). New York: Oxford University Press. (Originalmente publicado em 1940).

Carneiro, B. V. (2008). Propriedades psicométricas da OAS - Observer Alexithymia Scale: versão brasileira. Tese de Doutorado, Pontifícia Universidade Católica de Campinas, Campinas.

Corcos, M., \& Jeammet, P. (2000). Conduites de dépendance à l'adolescence: aspects étiopathogéniques et cliniques. Encyclopédie Médico-Chirurgicale, 20, 370-400.

Guimarães L. P. M, \& Yoshida, E. M. P. (2008). Doença de Crohn e Retocolite Ulcerativa Inespecífica: Alexitimia e adaptação. Psicologia: Teoria e Prática, 10, 52-63.

Haviland, M. G., Shaw, D. G., Cummings, M. A., \& MacMurray, J. P. (1988). Validation of the Toronto Alexithymia Scale with substance abusers. Psychotherapy and Psychosomatics, 50, 81-87.

Haviland, M. G., \& Reise, S. P. (1996). A California Q-set Alexithymia Prototype and its relationship to ego-control and ego-resiliency. Journal of Psychosomatic Research, 41, 597-608.

Haviland, M. G., Warren, W. L., \& Riggs, M. L. (2000). An observer scale to measure alexithymia. Psychosomatics, 41, 385-392.

Jula, A., Salminen, J. K., \& Saarijãrvi, S. (1999). Alexithymia: A facet of essential hypertension. Hypertension, 33, 1057-1061.

Krystal, J. H., Giller, E. L., \& Cicchetti, D. V. (1986). Assessment of alexithymia in posttraumatic stress disorder and somatic illness: Introduction of a reliable measure. Psychosomatic Medicine, 48, 84-94.

Lane, R. D., Sechrest, L., Riedel, R., Shapiro, D. E., \& Kaszniak, A. W. (2000). Pervasive emotion recognition deficit common to alexithymia and repressive coping style. Psychosomatic Medicine, 62, 492-501.

Laplanche, J., \& Pontalis, J. B. (1970). Vocabulário da psicanálise. Lisboa: Moraes Editores. (Originalmente publicado em 1967). 
Larsen, K. J., Brand, N., Bermond, B., \& Hijman, R. (2003). Cognitive and emotional characteristics of alexithymia: A review of neurobiological studies. Journal of Psychosomatic Research, 54, 533-541.

Lesser, I. M. (1981). A review of the alexithymia concept. Psychosomatic Medicine, 43, 531-541.

Lipp, M. E. N., Alcino, A. B., Bignotto, M. M., \& Malagris, L. E. N. (1998). O treino de controle de estresse para hipertensos: uma contribuição para a medicina comportamental. Estudos de Psicologia (Campinas), 15, 59-66.

Luminent, O., Bagby, R. M., \& Taylor, G. J. (2001). An evaluation of absolute and relative stability of alexithymia in patients with major depression. Psychotherapy and Psychosomatics, 70, 254-260.

Maciel, M. J. N., \& Yoshida, E. M. P. (2006). Avaliação da alexitimia, neuroticismo e depressão em dependentes de álcool. Avaliação Psicológica, 5, 43-54.

Mann, L. S., Wise, T. N., Trinidad, A., \& Kohanski, R. (1995). Alexithymia, affect recognition, and five factors of personality in substance abusers. Perceptual and Motor Skills, 81, 35-40.

Martínez-Sánchez, F., Ato-Garcia, M., \& Ortiz-Soria, B. (2003). Alexithymia, state or trait? The Spanish Journal of Psychology, 6, 51-59.

Marty, P., \& M'Uzan, M. (1994). O pensamento operatório. Revista Brasileira de Psicanálise, 28, 165-174. (Originalmente publicado em 1963)

Mc Dougall, J. (1982). Alexithymia: A psychoanalytic viewpoint. Psychotherapy and Psychosomatics, 38, 81-90.

Messer, S. B., \& Warren, C. S. (1995). Models of brief psychodynamic therapy. New York: Guilford Press.

Morrison, S. L., \& Pihl, R. O. (1989). Psychometrics of the SchallingSifneos and Toronto Alexithymia Scales. Psychotherapy and Psychosomatics, 51, 83-90.

Oliveira, M. R. L. G. de (2001). Alexitimia na algia pélvica crônica. Tese de Doutorado, Universidade de Campinas, Campinas.

Peçanha, D. L. (1998). Contribuições teóricas do Instituto de Psicossomática de Paris. Cadernos de Psicologia, 4, 129-137.

Pedinielli, J. L., \& Rouan, G. (1998). Concept d'alexithymie et son interêt en psichosomatique. Encyclopédie Médico-Chirurgicale, 20,370-400.

Pregnolatto, A. P. F. (2005). Alexitimia e sintomas psicopatológicos em pacientes com insuficiência renal crônica. Dissertação de Mestrado, Pontifícia Universidade Católica de Campinas, Campinas.

Purves, D., Augustine, G. J., Fitzpatrick, D., Katz, L. C., LaMantia, A., \& McNamara, J. O. (1997). Emotions. Em D. Purves, G. J. Augustine, D. Fitzpatrick, L. C. Katz, A. LaMantia \& J. O. McNamara (Orgs). Neuroscience (pp. 513-527). Massachusetts: Sinauer.

Sifneos, P. (1977). Psychothérapie brève et crise emotionelle. (G. Hougardy \& D. Luminet, Trads.) Bruxelles: Pierre Mardaga. (Trabalho original publicado em 1972)

Sifneos, P. E. (1991). Affect, emotional conflict, and deficit: An overview. Psychotherapy and Psychosomatics, 56, 116-122.
Sifneos, P. E., Apfel-Savitz, R., \& Frankel, F. H. (1977). The phenomenon of Alexithymia: Observations in neurotic and psychosomatic patients. Psychotherapy and Psychosomatics, 28, 47-57.

Silva, A. F. R. D., \& Caldeira, G. (1992). Alexitimia e pensamento operatório, a questão do afeto na psicossomática. Em J. Mello Filho (Org), Psicossomática hoje (pp. 113-118). Porto Alegre: Artes Médicas.

Taieb, O., Corcos, M., Loas, G., Speranza, M., Guilbaud, O., Perez-Diaz, F., Halfon, O., Lang, F., Bizouard, P., Venisse, J. L., Flament, M., \& Jeammet, P. (2002). Alexithymie et dépendance à l'alcool. Annales de Médecine Interne, 153, 1S51-1S60.

Taylor, G. J. (1984). Alexithymia: Concept, measurement, and implications for treatment. The American Journal of Psychiatry, 141, 725-732.

Taylor, G. J. (1990). La pensée opératoire et le concept d'alexithymie. Reviste Française de Psychanalyse, 3, 669-784.

Taylor, G. J. (2000). Recent developments in alexithymia theory and research. Canadian Journal of Psychiatry, 45, 134-142.

Taylor, G. J., Ryan, D., \& Bagby, R. M. (1985). Toward the development of a new self-report alexithymia scale. Psychotherapy and Psychosomatics, 44, 191-199.

Taylor, G. J., \& Bagby, R. M. (2004). New trends in alexithymia research. Psychotherapy and Psychosomatics, 73, 68-77.

Torres, A. R., \& Crepaldi, A. L. (2002). Sobre o transtorno de pânico e a hipocondria: uma revisão. Revista Brasileira de Psiquiatria, 24, 144-151.

Wiethaeuper, D., Balbinotti, M. A. A., Pelisoli, C., \& Barbosa, M. L. L. (2005). Estudos da consistência interna e fatorial confirmatória da ETA-20 com estudantes brasileiros. Revista Interamericana de Psicologia, 39, 1-17.

WHO (2004). Neuroscience of psychoactive substance use and dependence. Geneva: World Health Organization.

Yoshida, E. M. P. (2000). Toronto Alexithymia Scales: precisão e validade da versão em português. Psicologia: Teoria e Prática, 2, 59-73.

Yoshida, E. M. P. (2007). Validade da versão em português da Toronto Alexithymia Scale - TAS em amostra clínica. Psicologia: Reflexão e Crítica, 20, 389-396.

Yoshida, E. M. P., \& Silva, F. R. S. (2007). Escala de Avaliação de Sintomas-40 (EAS-40): validade e precisão em amostra de universitários. Revista de Psicologia Escolar e Educacional, 11, 89-99. 